\title{
Online Security Assessment with Load and Renewable Generation Uncertainty: the iTesla Project Approach
}

\author{
M. H. Vasconcelos ${ }^{1,2}$, L. M. Carvalho ${ }^{1,3}$, J. Meirinhos ${ }^{1}$, \\ N. Omont ${ }^{4}$, P. Gambier-Morel ${ }^{4}$, G. Jamgotchian ${ }^{4}$ \\ ${ }^{1}$ INESC TEC - Porto, Portugal; ${ }^{2}$ FEUP - Porto, Portugal; \\ ${ }^{3}$ U. Lusíada - V. N. Famalicão, ${ }^{4}$ RTE - Paris, France \\ mhv@fe.up.pt; nicolas.omont@ @rte-france.com
}

\begin{abstract}
The secure integration of renewable generation into modern power systems requires an appropriate assessment of the security of the system in real-time. The uncertainty associated with renewable power makes it impossible to tackle this problem via a brute-force approach, i.e. it is not possible to run detailed online static or dynamic simulations for all possible security problems and realizations of load and renewable power. Intelligent approaches for online security assessment with forecast uncertainty modeling are being sought to better handle contingency events. This paper reports the platform developed within the iTesla project for online static and dynamic security assessment. This innovative and open-source computational platform is composed of several modules such as detailed static and dynamic simulation, machine learning, forecast uncertainty representation and optimization tools to not only filter contingencies but also to provide the best control actions to avoid possible unsecure situations. Based on High Performance Computing (HPC), the iTesla platform was tested in the French network for a specific security problem: overload of transmission circuits. The results obtained show that forecast uncertainty representation is of the utmost importance, since from apparently secure forecast network states, it is possible to obtain unsecure situations that need to be tackled in advance by the system operator.
\end{abstract}

Index Terms--Online Static/Dynamic Security Assessment, Uncertainty Modeling, Machine Learning, Renewable Power, High Performance Computing.

\section{INTRODUCTION}

The operation of a transmission grid involves the need to check ahead that the proper functioning of the system is unaffected by any plausible contingency. In Europe, Regional Security Coordination Initiatives (RSCI), like Coreso, achieve such security analyses from two days ahead to operations. Generally, the analyses consist on the verification of the "N-1" rule and involves the deterministic forecasting of the grid state based on either controlled or uncontrolled variables. Coreso does not perform the forecast as these are received from individual Transmission System Operators (TSO) and merged into one common grid forecast.

This research has received funding from the European Union $7^{\text {th }}$ Framework Programme under grant agreement $n^{\circ} 283012$ (www.iteslaproject.eu) and by the ERDF - European Regional Development Fund through the Operational Programme for Competitiveness and Internationalisation - COMPETE 2020 Programme within project «POCI-010145-FEDER-006961», and by National Funds through the FCT - Fundação para a Ciência e a Tecnologia (Portuguese Foundation for Science and Technology) as part of project «UID/EEA/50014/2013».

\author{
D. Cirio $^{5}$, E. Ciapessoni ${ }^{5}$, A. Pitto ${ }^{5}$, \\ I. Konstantelos ${ }^{6}$, G. Strbac ${ }^{6}$, M. Ferraro ${ }^{7}$, C. Biasuzzi ${ }^{7}$ \\ ${ }^{5}$ RSE - Milano, Italy; ${ }^{6}$ Imperial College - London, United \\ Kingdom; ${ }^{7}$ Quinary - Milano, Italy
}

The increasing deployment of Renewable Energy Sources (RES) increases the forecast uncertainty and TSOs are incentivized to operate the system closer to its limits without jeopardizing the risk level. Traditionally, online dynamic security assessment (SA) has been carried out by using numerical methods that solve the algebraic and the differential equations of the power system components' models [1] for plausible contingency events. Such methods are computationally intensive, which makes the online scanning of potential dynamic problems for real power systems an intractable task. Direct methods via Lyapunov or Energy Functions [2]-[4] have been proposed to overcome the computational burden of numerically simulating the evolution of the system following a disturbance. These methods are mathematically sound but have limited application (transient stability). More recently, machine learning-based methods were proposed as an alternative way to automatically infer the security level of the system following a disturbance. These techniques are trained offline for a wide-range of operation conditions and security problems. The parametrization of these methods is a computationally intensive task that requires the static and dynamic simulation of the system for each credible contingency and operation condition. After appropriately parametrized, these methods have the advantage of requiring little to no computational effort to infer the security of system states within a margin of error, requiring only the state of a limited set of variables as input. A good parametrization procedure makes sure that the classification error is minimal for operation conditions that might occur in the future. Amongst numerous machine learning methods available in the literature, Decision Trees (DTs) [5]-[7], Neural Networks [8]-[10], and Fuzzy Inference Systems [11][12], are the ones mostly used for online SA.

More recently, researchers have proposed holistic frameworks [13]-[15] for SA that combine offline and online applications for assessing different phenomena (e.g. steadystate violations, frequency stability, transient stability, voltage stability, small-signal stability) with uncertainty. These frameworks aim at bridging the gap between risk-based methods and traditional deterministic approaches, by introducing the concepts of probability and impact associated 
to the contingencies as well as the uncertainty of the energy consumption forecast and of the RES power production. The ultimate goal of these frameworks is to devise overall architectures for online SA integrating probabilistic and deterministic concepts in a unified environment.

This rationale has triggered the developments of the iTesla project $^{1}$ which is co-funded by the European Commission Framework Program 7 (EC FP7). The project required the development of an online security analysis platform able (a) to cope with European-wide grid models, (b) to account for uncertainty and its impact on the implicit or explicit margins needed to ensure the wanted security level (c) to handle the curative remedial actions needed to reduce the margins that are necessary to face contingencies, and (d) to achieve both static and dynamic simulations in order to ensure the accurate evaluation of the security of the system when operated closer to its limits. An important prerequisite is the persistence of grid element identifiers, throughout time and also between forecast and snapshot (SN) operating scenarios, so that it is possible (a) to assess the uncertainties at nodal level and (b) to apply specific curative remedial actions stored into a catalogue associated with the grid model. This property can be ensured thanks to the Common Grid Model Exchange Standard (CGMES), which should be deployed throughout European TSOs in the years to come so that the operational deployment of the iTesla platform will be made possible.

Validation tests have been defined and implemented for different test cases. An important step of the validation process is to make sure that the iTesla platform is able to produce outputs consistent with TSO operational knowledge. Overloaded lines after the occurrence of a contingency are commonly simulated and studied on the French network. Therefore, this security problem was selected to perform the first validation tests and the results are reported in this paper. This paper is structured as follows: first, an overview of the iTesla platform is presented in Section II; then, Section III describes the results obtained from the application of the iTesla platform to the online SA of a contingency in the French network; finally, Section IV presents general conclusions and points directions towards future work.

\section{THE ITESLA PlATFORM}

The iTesla platform is an open source and interoperable tool that supports operators in the SA of forecast situations from several hours ahead of operation up to near real time. Recommendations are also provided in terms of efficiency of curative remedial actions when they are needed. The iTesla platform is able to take into account the uncertainties affecting power injections, such as non-programmable RES and loads, and the dynamic behavior of the grid, thanks to a filtering approach that takes advantage of machine learning techniques. The computations are performed with two complementary workflows, namely the offline and the online workflows (see Fig. 1). The offline workflow builds (1) security rules and (2) uncertainty models for use in the online workflow: the security rules are applied to plausible states in the "uncertainty domain" of the forecast under analysis, to identify the

${ }^{1} \underline{\text { www.itesla-project.eu }}$ contingencies for which control actions are needed, while limiting the number of accurate network simulations to be performed online. Both workflows include different computation modules, each fulfilling a specific technical function such as power flow (PF) computation or time-domain simulation.

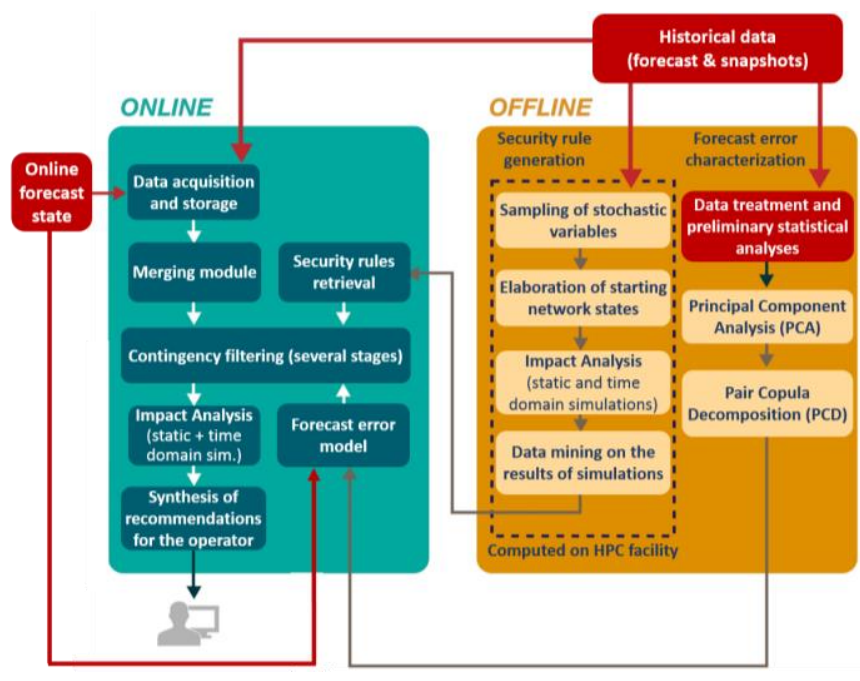

Fig. 1. The iTesla platform architecture: the online and offline workflows

\section{A. Offline Computation of Security Rules}

The offline workflow is expected to be executed once every week in order to update the security rules with the latest information concerning possible system topological changes, general weather patterns, etc. As such, the set of security rules computed must be generic enough to enable classification of unseen operating conditions likely to occur over a span of a week. To achieve this goal, the offline platform is based on the triptych of anticipate-analyze-classify. The overall idea is to use simulations in order to sample the system's security boundary. By extrapolating the information of historical data, predictive capability can be constructed that will support online operation. To this end, the iTesla platform generates a population of anticipated network states. The postcontingency security of each state is then determined against a set of credible contingencies. Machine learning is finally applied to enable inference to other network states not seen in this training process. This way, generalizable security rules with high predictive capability can be constructed. These three steps are explained in more detail below.

The first step of the offline workflow is to build a large population of network states that are likely to occur. Historical measurements from similar periods are used to inform a model that generates a very large number of scenarios. This process is split in two parts. Firstly, scenarios of different stochastic variable realizations (i.e. all variables beyond the operator's control such as demand and wind power production) are built. This is done by fitting a sophisticated statistical model, based on Principal Component Analysis (PCA) and Pair Copula Decomposition (PCD) with C-vines, to relevant historical data [16]. The constructed model is subsequently sampled at a high density. Each generated scenario is then transformed into a complete network state where all state variables (e.g. dispatch levels, substation topology, PF etc.) are defined. For this 
purpose, a state-of-the-art tool [17] comprised of four hierarchical optimization levels is run to infer the operator's actions. The generated scenarios are consistent with what has been observed historically, but also capable of exploring marginal cases that have rarely occurred in the past.

The second and most resource-intensive step is to analyze the impact of each credible contingency across all anticipated network states. This is done by carrying out a static and timedomain simulation and investigating the impact of a particular asset failure event. The impact of a contingency on a pre-fault state is summarized through post-fault security indices that have been developed to detect steady-state and dynamic problems such as over/under-voltage, overloads, angular, small signal and voltage instability [18][19]. Note that this step can be a computational bottleneck due to the very large number of simulations required (1 million simulations arise when analyzing 1000 contingencies across 1000 network states). The iTesla platform's excellent scaling capability has been verified in experiments using up to 10,000 cores [20].

The third and final step consists of applying a suitable machine learning algorithm. The results of millions of simulations are compressed into a set of security rules that are used online to classify unseen network states as secure or unsecure against specific contingencies in a very fast way. Note that a security rule is obtained for each contingency and security phenomenon (overloads, transient stability, etc.). Security rules are expressed as DTs [21] which have the advantage of being easily interpretable and suitable for integration as linear constraints into optimization tools.

\section{B. Offline Computation of Uncertainty Models}

Loads and RES forecast uncertainties are taken into account by defining probabilistic models of the forecast error i.e. of the difference between forecast values (e.g. evaluated on the day ahead) and values occurring in real operation SN. Forecast error models are synthesized, based on historical data series of these forecasts and SNs. These models account for: (a) Dependences between injections. For instance, several injections may be affected by similar errors, when the forecast is based on weather variables (sun radiation, wind; temperature). (b) Forecast values themselves (e.g. in case of very sunny or very cloudy weather, uncertainty on PV production will be small, and vice versa in case of partially clouded sky).

The uncertainty modelling problem can be very complicated due to the high number of stochastic injections. Moreover, probability distributions of forecast errors are nonGaussian and their dependences are non-linear. The offline platform applies an approach based on PCA and PCD similar to the one discussed in subsection II.A to build a reasonable statistical model of forecast errors. Again, some computational time intensive operations on large historical datasets (e.g. matrix inversions) are performed offline to pre-compute some quantities to be used in the on-line platform.

\section{Online Security Assessment}

\section{1) Monte Carlo-Like Approach}

Monte Carlo-Like Approach (MCLA) performs the sampling of the uncertainty domain around the forecast state, and then checks each new sampled state against the security rules. MCLA receives as input the forecast states with an associated uncertainty model computed offline, the list of contingencies and the security rules computed by the offline workflow. New plausible states are computed online from the forecast state by sampling the stochastic injections (loads and RES) and adjusting the conventional generation accordingly. Sampling "conditions" the forecast error model to the current forecast under analysis accounts for the dependence among conditioned variables. The adopted sampling technique limits the on-line computation burden by exploiting several quantities computed offline (see subsection II.A), Nataf transformation [22] and the properties of Gaussian conditional multivariates. This makes the technique suitable for online applications dealing with large sets of stochastic variables. The output of MCLA is the security assessment of each evaluated state with respect to the analyzed contingencies.

\section{2) Control Module for Remedial Action Identification}

Curative remedial actions are investigated for all the \{state, contingency\} couples resulting from the previous step labelled as unsecure without it. Security-Constrained Optimal Power Flow (SC-OPF) techniques are involved in this step. The optimization module includes a solver dedicated to topological reconfiguration. Topology and the related curative actions are modelled through binary variables in the nonlinear optimization problem. Thanks to an innovative method, this module is able to find, in a fast way, the necessary curative remedial actions to avoid current limits violation. An important element in that approach is the fact that a limited number of control actions are considered. Indeed, the choice has to be made between control actions that have been selected by the dispatchers and which are dedicated to the specific couple \{contingency, violated constraints .

\section{3) Detailed Network Simulation}

Static and time-domain simulation is carried out for the cases that either are not solved by the control module, or may exhibit stability problems. In this case, the security evaluation is obtained by applying the indices that were used to build the security rules. Additional testing, by simulating the effectiveness of the control action proposed by the control module, is also an option implemented in the platform.

\section{4) Fuzzy Power Flow Approach}

PF methodologies based on the fuzzy set theory can have advantages over the traditional probabilistic approaches since the former are able to model empirical knowledge not related to event repetition such as "load between 10 and $12 \mathrm{MW}$ " or "generation around $50 \mathrm{MW}$ ". A fuzzy number is a connected set or range of possible values, where each possible value within that set has a weight between 0 and 1 . The aim of the Classic Fuzzy PF (FPF) [23] is to determine a membership function or, more simply, a fuzzy number for the bus voltage magnitude and angle as well as for the current and the apparent PF in the branches as a function of the fuzzy numbers specified for the active and reactive power injections.

The Classic FPF method was imbedded in the iTesla platform. It accepts triangular fuzzy numbers for the active and reactive power injections as inputs and computes triangular fuzzy numbers for the output variables of interest. 
The central value of the fuzzy number corresponds to the output obtained from the AC PF. The FPF module is meant to be run in parallel to the mainstream of the online process. Hence, this module can support validation of the contingency filtering for static phenomena and shows the modularity of the iTesla platform to integrate new functionalities. The FPF module can be run for several contingency scenarios and computes a Security Index (SI) based on the physical limits of the variables (e.g. maximum and minimum voltage magnitude) as follows

$$
\mathrm{SI}=\left(\int_{P B_{L}}^{P B_{H}} f(x) d x\right) /\left(\int_{-\infty}^{+\infty} f(x) d x\right)
$$

where $f$ is the membership function and $P B_{L}$ and $P B_{H}$ are, respectively, the lower and upper physical limits of the variable under analysis. Note that the SI belongs to the interval $[0,1]$. If the security index is 1 , then the fuzzy number computed is completely within the interval defined by its physical limits. Alternatively, a SI of 0 indicates that the fuzzy number is completely outside the physical boundaries of the variable. If the SI takes a value within the interval $] 0,1[$, then there is a possibility that the boundaries might be violated: the greater the security index, the smaller the violation possibility.

\section{Computational Implementation}

A high-end software framework was developed to handle the heavy computations required by not-deterministic security analysis, especially the ones of the offline module in the case (not shown in the current article) where a dynamic simulator is used instead of a PF tool. The platform, whose core is in $\mathrm{Java}^{2}$, is modular so as to ease the distribution of computations, through a High Performance Computing (HPC) layer built on top of a Message Passing Interface (MPI), and the replacement of modules (for example, the replacement of one PF tool by another). It provides common services such as data management, data conversion, data mining, task management and graphical user interfaces. MongoDB was used to store injection and withdrawal chronicles extracted from grid models and to compute statistical indicators like quantiles for uncertainty analysis. Moreover, the platform contains a data model for static and dynamic power system data (IIDM iTesla Internal Data Model) and several conversion services between this data model and external formats (UCTE-DEF, CIM Profile 1, Eurostag, PSS/E). As mentioned earlier, the scaling of computation time was tested up to 10,000 cores.

\section{CASE STUdY}

Several contingency scenarios in the French network were analyzed in order to validate the iTesla platform for the overload security problem. This analysis consisted on the simulation of short-circuits in a single or double $400 \mathrm{kV}$ line that lead to the line disconnection, which may create overload problems in the neighboring $225 \mathrm{kV}$ lines and, therefore, are monitored by RTE on a regular basis. This section presents the results obtained for one of these contingencies in order to illustrate the functionalities and extent of results provided by the iTesla platform.

\footnotetext{
${ }^{2}$ Code available under MPL v2 license on https://github.com/itesla/ipst
}

Simulations were performed in the EHV part of the French network, by assuming that the boundary nodes are connected to fictitious loads reflecting the flows in the interconnections and in the HV network. Hence, the French network was reduced to 1416 substations, 427 generators, 1952 loads, 168 shunts and about 1700 nodes depending on the situation. The stochastic variables comprise the active and reactive power injection of loads and RES totaling 3798 variables. The historical data used was Day-Ahead Congestion Forecast (DACF) and SN files, in CIM Profile 1 format, with persistent identifiers for equipment regardless of the time and type of file (DACF and $\mathrm{SN}$ ). The iTesla platform detects unsecure situations due to overload by comparing the post-contingency steady-state current in all the lines and transformers with its operational limits in the CIM file: if at least one limit is violated, then the situation is considered unsecure.

\section{A. Offline Computation of Security Rules}

\section{1) Methodology for DTs Parametrization/Evaluation}

The security rules obtained to detect overload problems in the French network were computed with the DATAmaestro® ${ }^{3}$ software that was integrated in the iTesla platform. For each contingency/security index pair, this module computes DTs with alternative values for the training parameters, namely the alpha $(\alpha)$ and the true threshold $(t T)$ parameters. The $\alpha$ value allows controlling the size of the tree, by defining the minimum statistical significance (i.e. 0/1 means never/always significant) required to split nodes. Usually the optimal value of $\alpha$ is quite independent of the learning set and of specific problems of the disturbance, such as type, location and size of the system [24]. According to [25], the optimum value for $\alpha$ lies between $[0.00005 ; 0.001]$. The $t T$ value defines how the security rule is generated from the DT structure. For not pure leaves, $t T$ defines the threshold for the proportion of secure learning states to produce a secure prediction.

For the parametrization/selection of the best DT for each contingency/security index pair, a quasi-automatic procedure was applied comprising the following steps: (a) change the training parameters of the DT; (b) DT training with the k-fold cross validation method [21] for a $20 \%$ test set size; (c) evaluation of the DT performance by analyzing the training misclassification errors, the DT structure visualization and the geographical visualization of the DT. From this procedure, a set of alternative DTs is obtained and the best DT is selected according to several criteria. The highest priority criterion is to maximize the accuracy of the DT to detect unsecure situations, which is achieved by minimizing the Missed Alarms (MA) (i.e. unsecure state/contingency pairs for which the DT provided a secure classification). Hence, the number of MA must be, at least, reduced to a controlled risk by minimizing the following indicator: Probability of $\mathrm{MA}=$ \#ucs/(\#ucs+\#scs), where \#ucs is the number of unsecure states classified secure and \#scs is the number of well classified secure states.

An additional important criterion is to maximize the efficiency of the DTs in detecting secure situations, which aims at maximizing the filtering capability of the online part.

\footnotetext{
${ }^{3}$ http://mydatamaestro.com/
} 
This is achieved by minimizing the False Alarms (FA) situations (i.e. secure state/contingency pairs for which the DT provided an unsecure classification) and also by maximizing the following mathematical indicator: DT filtering capability = (\#ucs+\#scs)/(\#states), where \#states is the number of states.

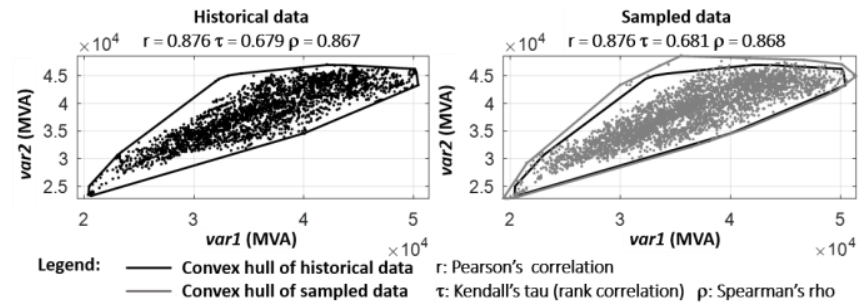

Fig. 2. Visual comparison between historical and sampled stochastic data

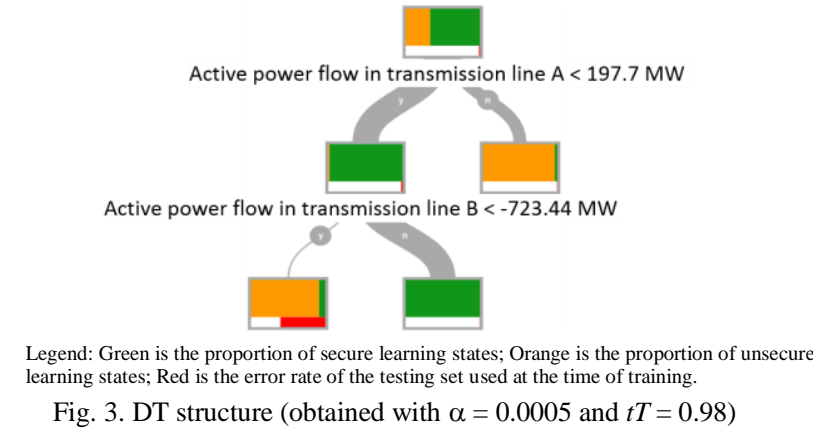

Avoiding DTs over-fitting to the learning states was also pursued in order to have a good generalization capability and obtain accurate estimations for unseen states. The over-fitting problem was controlled by: (a) not letting the testing set errors to be significantly higher than the learning set errors; (b) to avoid a tree structure that includes, in their splitting tests, operation conditions that have no relationship with the security problem under analysis. Finally, the DT parametrized was further evaluated with historical data not used for training to assess its generalization capability.

\section{2) Execution of the iTesla Platform}

The offline part of the iTesla platform was run by using the SN of January 2013 to sample stochastic variables and create realistic network states using optimization. Then, for each network state sampled, the post-contingency state was computed to produce a large data set of pre-analyzed states for the parametrization/selection of the best DTs. Finally, the DTs were applied to all the SN and DACF of February 2013 to assess the generalization capabilities of the DTs. The computations were run on $2 \times$ Intel(R) Xeon(R) CPU E5-2670 0 @ 2.60GHz processors (16 cores) and 128 GB of RAM.

\section{3) Validation of the Sampling of Stochastic Variables}

The iTesla platform contains a tool that allows a visual comparison between the historical SN used for sampling and the sampled states, in the form of plots on relevant sets of the stochastic variables. Good quality sampling is obtained when these plots reveal a similar dependence structures between historical SN and the sampled states. Fig. 2 presents some of the plots obtained for aggregated stochastic variables. The variable in $x$-axis of this figure, varl, represents the summation of the first half of the stochastic variables (from 1 to 1899 ), whereas the variable in the $y$-axis, var2, comprises the summation of the second half of stochastic variables (from 1900 to 3798). Note that the left plot represents the historical data and the right plot was obtained for the sampled data. For easier detection of outliers, these plots also present the convex hull of each dataset (in black for historical and in gray for sampled data).
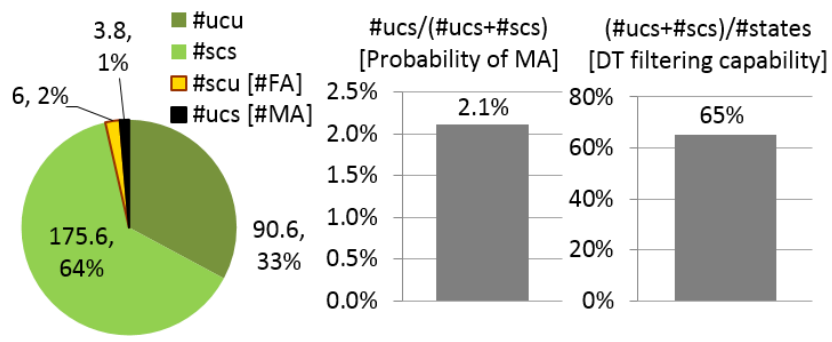

Legend: \#ucu = \#Unsecure classified Unsecure; \#scs = \#Secure classified Secure; \#scu = \#Secure classified Unsecure; \#ucs = \#Unsecure classified Secure

Fig. 4. k-fold cross validation statistics of the DT

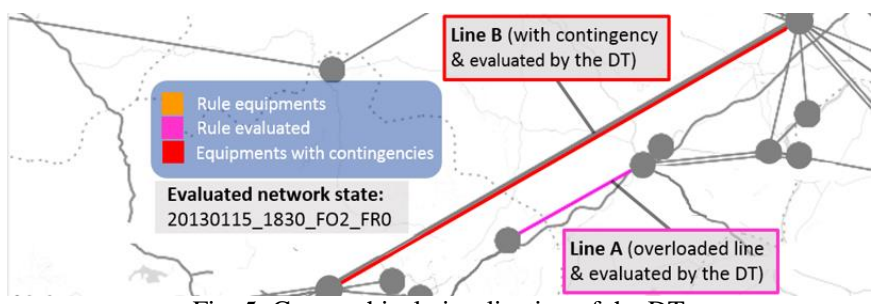

Fig. 5. Geographical visualization of the DT

The following statistics are also provided to assist the visual comparison: Pearson's correlation $(r)$; Kendall's tau rank correlation $(\tau)$ and Spearman's rho $(\rho)$. These plots show that between historical and sampled data: (a) the stochastic aggregated variables occupy roughly the same area of the state space; (b) the dependence structure is largely the same. Hence, the results presented illustrate that the generated sampled stochastic variables seem consistent with the input historical data $(\mathrm{SN})$.

\section{4) Decision Tree Validation Process}

The DT structure obtained for one of the analyzed contingencies is presented in Fig. 3. (short-circuit in a transmission line leading to its disconnection). This DT was generated with a data set of 1384 pre-analyzed network states, comprising $66 \%$ of secure states for the analyzed contingency. The obtained k-fold cross validation statistics for this DT are presented in Fig. 4. From these statistics, the obtained DT misclassification errors seem being reduced to a controlled risk. Namely, the obtained $\mathrm{k}$-fold cross validation errors comprise $1 \%$ of MA states and $2 \%$ of FA states, defining a probability of MA of $2.1 \%$ and a DT filtering capability of $65 \%$ (very close to the $66 \%$ of secure states in the data set).

The geographical visualization of the analyzed DT is presented in Fig. 5 (image anonymized due to confidentiality issues). This image shows the geographical location of the network equipment involved in the contingency (highlighted in red) and the equipment involved in the security rule (highlighted in pink for the equipment's used to classify a preselected network situation and in orange for the remaining ones). From this image, it was possible to verify that: (a) The first split of the DT defines a threshold for the active power flow in the transmission line that may become overloaded 
after this contingency (information obtained from TSO expertise); (b) The remaining split of the DT defines a threshold for the active power in the transmission line where the contingency was simulated. These results demonstrate that the operating conditions involved in the DT structure are strongly related with the analyzed security problem and, therefore, validate the quality of the DT.
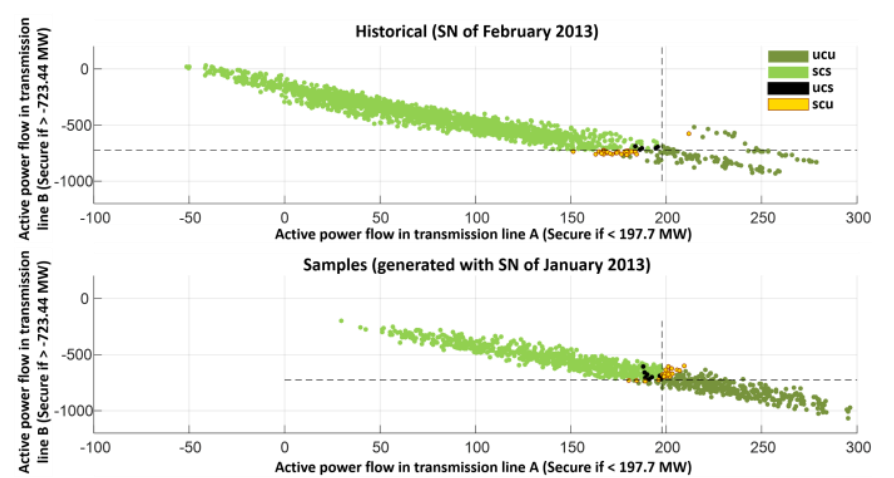

Fig. 6. 2D 4-color plots for the SN of February 2013

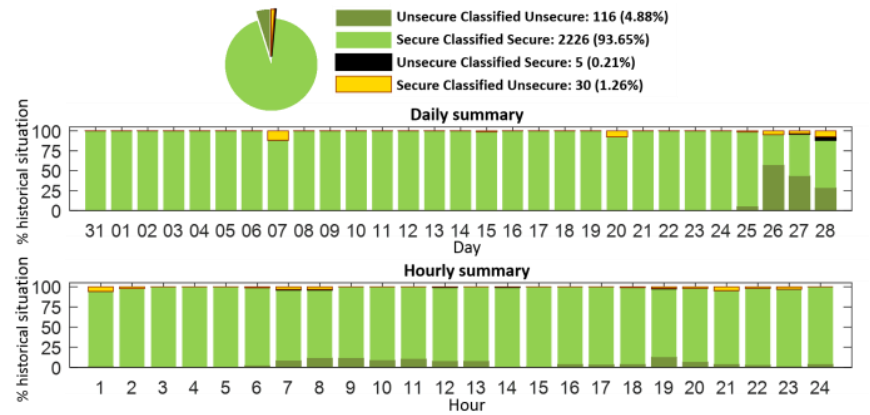

Fig. 7. Overall, daily and hourly performance for the SN of Feb/2013

\section{5) Decision Tree Generalization Capability}

The generalization capabilities of the best DT obtained for the selected contingency was used to classify the SN and DACF of February 2013. By comparing the DT classification results with the true state classification, it was possible to obtain the following statistics: \#ucu: number of well classified unsecure states; \#scs: number of well classified secure states; \#ucs: number of unsecure states classified secure (number of MA); \#scu: number of secure states classified unsecure (number of FA). Moreover, to help at visualizing the results of this statistical analysis, a 4-color 2D plot was created (see Fig. 6) which was obtained after using the DT depicted in Fig. 3 to classify the SN of February 2013 (plot labelled Historical). Fig. 6 also presents the same results for the states used to train the DT (plot labelled Samples).

The variables selected as axis of the 4-color 2D plot are the two most important attributes used by the DT to sort out the secure states from the unsecure ones. The color code used in this figure is the one also used in the pie chart of Fig. 4. The dashed lines correspond to the security thresholds defined by the DT. These plots allow to visualize the security domain and the relative distance of the ucu, scs, ucs and scu from the thresholds assigned to the variables of the DT. What is more, these plots allow to infer the appropriateness of the DT to classify new states by checking the relative location of these new states from the security domain. If the new states
(Historical plot) are outside the zone defined by the training states (Samples plot), we can postulate that the DT is not being used within its validity domain.

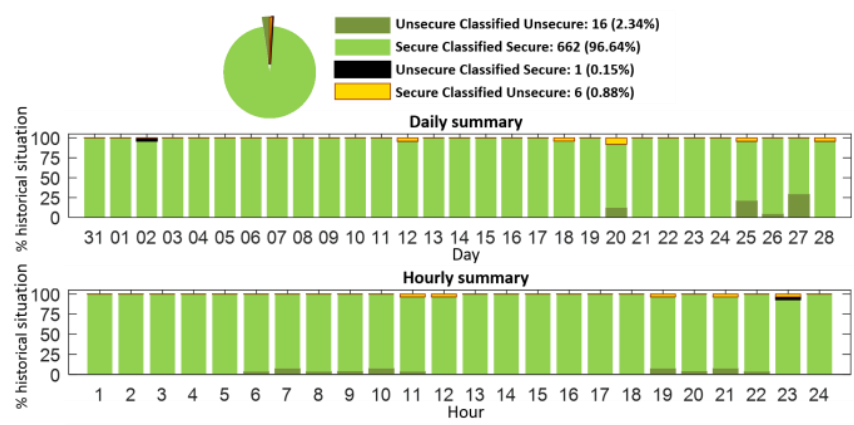

Fig. 8. Overall, daily and hourly performance for the DACF of Feb/2013

Fig. 6 shows that the set of historical $\mathrm{SN}$ is biased towards the secure area of the space, showing that February 2013 was considerably more secure than January 2013. Moreover, note that there is a low number of MA and FA in the SN of February 2013 and their location is near the security boundary. Hence, it can be deduced that this DT bears sufficient generalization capability to satisfactorily split the region of secure states from the region of unsecure ones. To complement the information in Fig. 6, Fig. 7 plots the overall, daily and hourly distribution of the correctly and incorrectly classified SN of February 2013. Note that only 5 MA are provided during the entire month, which proves the good performance of the DT for this particular contingency and security problem. Besides, from the daily summary, it is possible to realize that a few unsecure situations occurred in the end of February 2013. In order to evaluate the quality of the DT with more realistic conditions, the former analysis was also carried out for the DACF of February 2013. The overall, daily and hourly distribution of the FA and MA are presented in Fig. 8. These results indicate that this DT provides good generalization capabilities not only for SN but also for DACF.

\section{B. Online Security Assessement with Uncertainty}

\section{1) Monte Carlo Like Approach to detect state security}

The DT generated by the offline workflow of the iTesla platform was used to perform online SA of the analyzed contingency and security problem, including the uncertainties provided by the MCLA. For validation purposes, this procedure was applied to the DACFs of the 25 and 27 of February 2013, since these were the most unsecure days forecasted for this month (see Fig. 8). The forecast error model of stochastic variables was calculated by comparing the DACF of January 2013 with the SN of the same month. For validation purposes, the DT classification results were compared with the true state classification which was provided after the detailed simulation of the contingency analyzed. The MCLA results obtained for the DACFs of day 27 are summarized in Fig. 9. The top bar plot presents the DT statistics for each DACF forecasted for that day. The bottom column chart presents the DT statistics obtained for the same DACF, but now including the 100 states generated by the MCLA to model the uncertainty around the DACF. In order to better evaluate these results, a 2D 4-color plot was generated for the 100 samples and the DACF in the security domain of 
the DT. These plots are shown in Fig. 10 for four relevant situations in which: (a) the blue cross defines the location of the DACF (base case); (b) the dots describe the location of the MCLA samples (uncertainty).

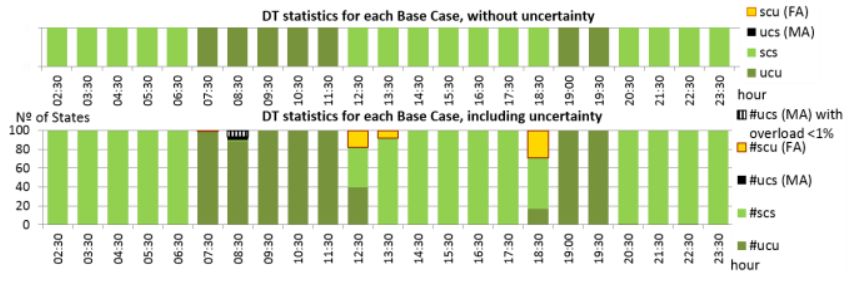

Fig. 9. DT statistics for all DACFs of $27 / \mathrm{Feb} / 2013$, including uncertainty
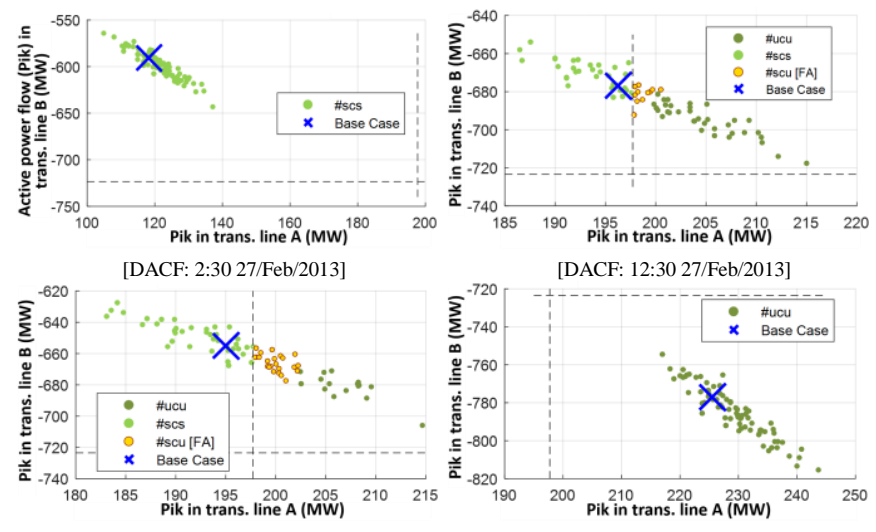

[DACF: 18:30 27/Feb/2013]

[DACF: 19:30 27/Feb/2013]

Fig. 10. Plots for some DACF of Feb/2013, including uncertainty

The results of Fig. 9 show that, when considering the forecast uncertainty, only a small number of states are FA (namely, for the DACF at 12:30, 13:30 and 18:30). Besides, there are some MA situations, namely at 8:30 which are, however, not critical since the DACF originating these MAs is, for most of its uncertainty domain, an unsecure situation and also because the overload problem is, in fact, smaller than $1 \%$ for most of the MAs. In addition, if the DACF is not located close to the security boundaries (see the DACF detailed in Fig. 10 at 2:30 and 19:30), all states sampled by MCLA share the same classification as the originating DACF. However, there are two DACFs in Fig. 10 (namely, at 12:30 and 18:30), in which it was possible to observe that, from an apparently secure situation (i.e. from a DACF with no overload problems) there is a considerable amount of unsecure states when the forecast uncertainty is taken into account. These results demonstrate the utmost need of including uncertainty when performing online SA.

\section{2) Fuzzy Power Flow as Additional Uncertainty Representation}

To validate the FPF approach, the FPF module integrated in the iTesla platform was applied for the same security problem and DACFs used to test the MCLA (see the previous section). The analysis of the FPF results was carried out aiming to compare the fuzzy uncertainty representation with the one provided by the MCLA, namely: (a) to detect if the states sampled by the MCLA are inside or outside the uncertainty region defined by the FPF for the two most important variables in the DT; (b) and to compare the classification of the DACF according to the Security Index (SI) defined for the FPF results. The fuzzy injections used as inputs, i.e. the uncertainty around each injection represented as a fuzzy triangular number, was obtained by using the mean and standard deviation extracted from the uncertainty distribution computed offline by the forecast error module. The lowest and highest values of the fuzzy number were computed from the central one, by subtracting and adding one standard deviation of the underlying uncertainty distribution.

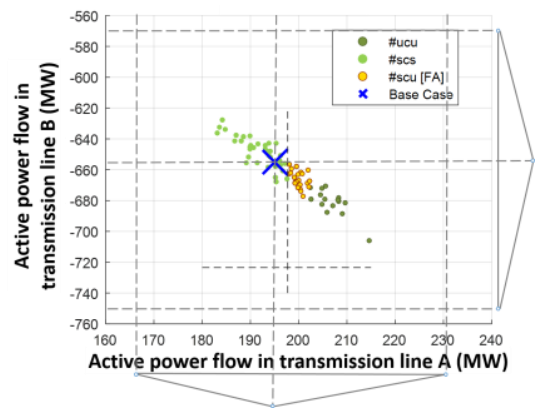

Fig. 11. Pre-contingency uncertainty defined by the MCLA and the FPF

To highlight the functionalities of the FPF module, the results obtained for one of DACFs analyzed are also presented, namely, for the 18:30 of the 27/Feb/2013 (see MCLA results in Fig. 9 and Fig. 10). The pre-contingency uncertainty characterization obtained by the MCLA as well as by the FPF module are presented in Fig. 11. This figure shows that all states sampled by the MCLA (dots) are included in the fuzzy region (external triangular shapes), which means that the results of FPF can be used as an upper bound for the MCLA uncertainty representation. Similar results were obtained for all the remaining tested DACFs of the French network.

The post-contingency fuzzy current, obtained for the transmission line that may become overloaded after the contingency leads to an SI of 0.6. This result indicates an unsecure DACF since the branch fuzzy current violates its maximum physical limit. This is consistent with the unsecure classification provided by the MCLA, since roughly $20 \%$ of the MCLA states were unsecure. The same consistency between secure/unsecure classifications with the MCLA and FPF were obtained for all tested DACFs. Therefore, it can be inferred that the FPF module tested is able to provide correct classifications for contingencies that result in overloaded equipment. Moreover, the fuzzy uncertainty representation was proven to be complementary and an upper bound to the probabilistic representation, which can be exploited to filter out contingencies before applying the MCLA.

\section{CONCLUSIONS}

This paper has presented the fundamentals of the platform developed, within the iTesla project, for improving the operation security of the network under load and renewable generation uncertainty. The ultimate goal of the platform developed is to support the decision making process during network operation from two-days ahead to real time based on three main features: (a) to provide a risk-based assessment taking into account different sources of uncertainties (e.g. load and renewable power generation) and contingency probability; (b) to perform accurate and fast online SA using time-domain simulations and machine learning techniques; (c) to provide operators with relevant proposals of preventive and curative 
actions to keep the system in a secure state. As a shared tool, the iTesla platform, whose code has been released opensource on Github (MPL 2.0 license), will help TSOs to address SA of their own system, of coordinated regional systems or of continental systems such as the whole PanEuropean network.

The iTesla platform was validated for a well-studied security issue in the French network: overload situations in transmission circuits. Several contingency events were analyzed, involving a short-circuit in a single or double 400 $\mathrm{kV}$ line resulting in line disconnection. These events can create overload problems in the neighboring $225 \mathrm{kV}$ lines, and therefore are regularly monitored by RTE. The results obtained have demonstrated the need to capture forecast uncertainty since unsecure operating situations can arise from apparently secure forecast network states. Alternative, uncertainty representations based on Fuzzy Set Theory have also been explored in the iTesla platform. The results obtained haven shown a complementariness between fuzzy and probabilistic forecast uncertainty representation that can be exploited for static contingency filtering and improve the computational performance of the platform.

Finally, the offline workflow will be improved so that security rules are generated with their corresponding validity domain. The online workflow uncertainty module will be tested in quasi-real time experimentations at RTE. It will be assessed that the measured flows on some important lines is in accordance with the forecasted flow distributions. Last but not least, curative remedial actions will be simulated -either statically or dynamically- before the security indexes are evaluated so as to avoid classifying as "unsecure" a situation and a contingency for which curative remedial actions are preplanned. It will improve the filtering capability and limit the call to the control module for remedial action identification to the most complex cases.

\section{ACKNOWLEDGMENT}

The authors would like to thank all iTesla partners for their contribution. We also gratefully acknowledge PRACE for awarding us access to resource CURIE based in France at Très Grand Centre de Calcul.

\section{REFERENCES}

[1] P. Kundur, Power System Stability and Control, McGraw-Hill Education, 1994.

[2] Hsiao-Dong Chang, Chia-Chi Chu and G. Cauley, "Direct stability analysis of electric power systems using energy functions: theory, applications, and perspective," Proc. of the IEEE, vol. 83, no. 11, pp. 1497-1529, Nov 1995.

[3] M. A. Pai and P. W. Sauer, "Stability analysis of power systems by Lyapunov's direct method," IEEE Control Syst. Mag., vol. 9, no. 1, pp. 23-27, Jan. 1989.

[4] Y. H. Song, L. Yao, P. Mao, and Y. Ni, "Fast estimation of transient stability limits by combining direct method with least squares technique," EPSR, vol. 48, no. 2, pp. 121-126, Dec. 1998.

[5] K. Sun, S. Likhate, V. Vittal, V. S. Kolluri and S. Mandal, "An Online Dynamic Security Assessment Scheme Using Phasor Measurements and Decision Trees," IEEE Trans. on Power Syst., vol. 22, no. 4, pp. 1935-1943, Nov. 2007.
[6] M. He, J. Zhang and V. Vittal, "Robust Online Dynamic Security Assessment Using Adaptive Ensemble Decision-Tree Learning," IEEE Trans. on Power Syst., vol. 28, no. 4, pp. 4089-4098, Nov. 2013.

[7] C. Liu et al., "A Systematic Approach for Dynamic Security Assessment and the Corresponding Preventive Control Scheme Based on Decision Trees," IEEE Trans. on Power Syst., vol. 29, no. 2, pp. 717-730, March 2014.

[8] K. W. Chan, A. R. Edwards, R. W. Dunn and A. R. Daniels, "On-line dynamic security contingency screening using artificial neural networks," IEE Proc. Generation, Transmission and Distribution, vol. 147, no. 6, pp. 367-372, Nov 2000.

[9] E. Abu-Al-Feilat, M. Bettayeb, H. Al-Duwaish, M. Abido and A. Mantawy, "A neural network-based approach for on-line dynamic stability assessment using synchronizing and damping torque coefficients," EPSR, vol. 39, no. 2, pp. 103-110, Nov. 1996.

[10] N. Amjady, "Dynamic voltage security assessment by a neural network based method," EPSR, vol. 66, no. 3, pp. 215-226, Sep. 2003.

[11] J. M. Gimenez Alvarez and P. E. Mercado, "Online Inference of the Dynamic Security Level of Power Systems Using Fuzzy Techniques," IEEE Trans. on Power Syst., vol. 22, no. 2, pp. 717-726, May 2007.

[12] J. M. Gimenez Alvarez and P. E. Mercado, "A new approach for power system online DSA using distributed processing and fuzzy logic," EPSR, vol. 77, no. 2, pp. 106-118, February 2007.

[13] Y. Xu, Z. Y. Dong, Z. Xu, K. Meng and K. P. Wong, "An Intelligent Dynamic Security Assessment Framework for Power Systems with Wind Power," IEEE Trans. on Industrial Informatics, vol. 8, no. 4, pp. 995-1003, Nov. 2012.

[14] E. Ciapessoni, D. Cirio, S. Grillo, S. Massucco, A. Pitto and F. Silvestro, "An Integrated Platform for Power System Security Assessment Implementing Probabilistic and Deterministic Methodologies," IEEE Syst. Journal, vol. 7, no. 4, pp. 845-853, Dec. 2013.

[15] J. P. Deane, F. Gracceva, A. Chiodi, M. Gargiulo, B. P. Ó. Gallachóir, "Assessing power system security. A framework and a multi model approach," Int. Journal of Elect. Power \& Energy Syst., vol. 73, pp. 283-297, Dec. 2015.

[16] M. Sun, I. Konstantelos, S. Tindemans and G. Strbac, "Evaluating Composite Approaches to Modelling High-Dimensional Stochastic Variables in Power Systems", to be presented at PSCC, Genoa, 2016.

[17] M. Ruiz, J. Maeght, A. Marié, P. Panciatici and A. Renaud, "A progressive method to solve large-scale AC Optimal Power Flow with discrete variables and control of the feasibility," in Proc. PSCC, Wroclaw, 2014.

[18] F.R.S. Sevilla and L. Vanfretti, "Static stability indexes for classification of power system time-domain simulations," in Proc. IEEE PES ISGT 2015, pp. 1-5, Washington, 2015.

[19] L. Vanfretti and F.R.S Sevilla, "A three-layer severity index for power system voltage stability assessment using time-series from dynamic simulations," in Proc. IEEE ISGT Europe 2014, Istanbul, pp. 1-5, Oct. 2014.

[20] I. Konstantelos, G. Jamgotchian, S.H. Tindemans, P. Duchesne, S. Cole, C. Merckx, G. Strbac and P. Panciatici, "Implementation of a Massively Parallel Dynamic Security Assessment Platform for LargeScale Grids", IEEE Trans. on Smart Grid, under review, submitted Nov. 2015.

[21] L. Breiman, H. F. Friedman, R. A. Olshen, and C. J. Stone, Classification and Regression Trees, Wadsworth and Brooks, Monterey, CA, USA, 1984.

[22] P-L. Liu, A. Der Kiureghian, "Multivariate Distribution Models with Prescribed Marginals and Covariances," Probabilistic Engineering Mechanics, Vol. 1, No. 2, pp. 105-112, 1986.

[23] V. Miranda, M. Matos and J. T. Saraiva, "Fuzzy load flow-New algorithms incorporating uncertain generation and load representation," in Proc. PSCC, pp. 621-627, Aug. 1990.

[24] L. Wehenkel and M. Pavella, "Decision tree approach to power systems security assessment, Int. Jour. of Electrical Power \& Energy Syst., vol. 15, no. 1, pp. 13-36, Feb.1993.

[25] L. Wehenkel, M. Pavella, "Decision trees and transient stability of electric power systems, Automatica, vol. 27, no. 1, pp. 115-134, Jan. 1991 